\title{
Study the Physical Properties of Medium Density Fiberboard Utilized by Hybrid Mixture of Efb Fiber and Kenaf Fiber
}

\author{
Amamer Redwan ${ }^{1,2^{*}}$, Khairiah Haji Badri ${ }^{1}$ and Azizah Bahrum ${ }^{1}$ \\ ${ }^{1}$ School of Chemical Sciences and Food Technology, Faculty of Science and Technology. \\ Universiti Kebangsaan Malaysia, 43600 UKM Bangi, Selangor, Malaysia. \\ ${ }^{2}$ Department of Chemistry, Faculty of Science, Bani Waleed University, Bani Waleed, Libya. \\ *Corresponding author: amamer1982@yahoo.com.
}

\begin{abstract}
In this study, a new hybrid medium density fiberboard was formulated by utilizing a fiber mixture composing of 50:50 empty fruit bunch and kenaf fiber coupled with palm-based prePolyurethane functioning as a binder. Kenaf as well as Empty fruit bunch fibers had a size range of 300 to $500 \mu \mathrm{m}$. Aluminum trihydroxide was used as the fire retardant at loading percentage of 0,5 , 10 , and $15 \mathrm{wt} \%$ of total mass of the matrix. Water absorption property for hybrid-MDF showed a different trend in water absorption capability and this can be correlated to the strong dependence on the interface adhesion between fiber and the polymer matrix. The water absorption decreased as the loading level of Aluminum trihydroxide increased $(6.5-2.3 \%)$ due to that fibers are held to each other through the interfacial bonding in the presence of Aluminum trihydroxide.
\end{abstract}

[DOI: $\underline{10.22401 / A N J S .22 .2 .04]}$

Keywords: pre-polyurethane; medium density fiberboard; water absorption; flame retardant.

\section{Introduction}

Although there are some provincial insufficiencies of wood-based fibers in some places, lignocellulosic fiber is still adequate and there is no extensive shortage of fiber [1]. Legitimate wood-based raw materials in African and Asian nations are in high demand, partly due to strong economic growth in Africa and Asia. Wood-based biomass is limited and costly for producers of bio-energy, pulp and paper, wood-based composite, fiberboards and timber [2]. Besides, the ever-increasing environmental concerns related to climate changes, wildlife diversity, biomass production, biological concerns and environmental awareness have directed impact to opt for an alternate source for fiber. As such, harvesting annual plant materials seems to be a promising possibility as an alternative option for lignocellulosic fiber composites [3]. Year-round harvest crops for example, jute, hemp, flax, corn, bamboo, kenaf and bagasse have been tested for the production of nonwood molecule and fiberboards [4]. Mediumdensity fiberboard (MDF) has a specific gravity around 0.50 to 0.80 (density of 500 to $800 \mathrm{~kg} / \mathrm{m} 3$ ). Fiberboard with specific gravity more than 0.80 (density higher than $800 \mathrm{~kg} / \mathrm{m} 3$ ) is called a hardboard (ASTM
D1554-1986) [5]. Generally, fiberboards work as insulator, protection panel, and covering material in building and construction industries whereby flat sheets of moderate strength are needed. The furniture industry provides the most prominent market for fiberboard [6]. Polyurethane is a high molecular weight polymer based on the polyaddition of poly functional hydroxyl and isocyanate. Isocyanate and polyol are combined together using suitable catalysts and other polyurethane additives [7]. The urethane prepolymer produced has two isocyanate groups on both ends of urethane prepolymer with urethane bonds. Prepolymers are regularly produced with a mole proportion of 2 moles of diisocyanate to 1 mole of polyol [8]. Medium-density fiberboard (MDF) is generally utilized in transportation, furniture, decoration, construction and other commercial enterprises because of its moderate density, good mechanical and physical properties and cheap [9]. Nevertheless, the limitation of MDF as building material is its flammability and smoke, fire retardant materials have been developed and used in building materials [10]. This study will investigate the impact of adding flame retardant (ATH) on the physical properties of hybrid medium-density fireboard. 


\section{Experimental}

\section{Materials And Preparation}

The palm-based polyol (PKO-p) was supplied by UKM Technology Sdn Bhd through its pilot plant at MPOB/UKM Station, Pekan Bangi Lama, Malaysia. The crude 4,4-methylene diphenyldiisocyanate (MDI), (Cosmonate M-200) was purchased from Cosmo-polyurethane, Port Klang, Malaysia. Acetone was supplied by Merck Sdn Bhd, Shah Alam, Malaysia. Polyethylene glycol (PEG200 with molecular weight of $200 \mathrm{Da}$ ) was purchased from Fluka Chemie Sdn. Bhd. Oil palm empty fruit bunch fiber (EFB) was obtained from Syarikat Seri Ulu Langat, Banting, Malaysia. Aluminium hydroxide (ATH) was manufactured by Fluka, Switzerland.

Dried Kenaf fibers (KF) and EFB fibers were refined using Ika Werke MF10 heavyduty grinder and then sieved to obtain sizes of $300-500 \mu \mathrm{m}$. The resin was prepared by mixing PKO-p and PEG200 in acetone (20 wt \%). The crude MDI was also mixed with acetone $(20 \mathrm{wt} \%)$. Both mixtures were agitated separately in small beakers at $200 \mathrm{rpm}$ for $60 \mathrm{~s}$ at room temperature. The amount of PKO-p and MDI used were at a ratio of 1:2. This formed the prepolymerised polyurethane resin (Pre-PU).

The hybrid medium-density fiberboard (MDF) was produced by mixing ATH and hybrid EFB/KF (ratio 50:50) with pre-PU. The ratio of pre-PU to EFB was at 35:65. The flame retardant (ATH) was mixed with the pre-PU at varying amount of 5, 10, and $15 \%$ (by weight) prior to blending with KF for $60 \mathrm{~s}$ and hot pressed at $56^{\circ} \mathrm{C}$ for $9 \mathrm{~min}$ at $760 \mathrm{MPa}$. The MDF was conditioned at room temperature for $24 \mathrm{~h}$ and then cut to desired size using Seppach circular saw model TS400IS for further characterizations.

\section{Characterization}

Water absorption (WA) test was also conducted onto the MDF. The effect of EFB, KF fibers and ATH onto the swelling behavior and degree of water absorption of the samples was observed. The specimen was immersed into the distill water for seven days at room temperature and the reading was taken every $24 \mathrm{~h}$. Water absorption was determined according to ASTM D570-8 (Standard test method for water absorption of plastics). The weight percentage gain (WPG) was calculated using equation below:

$$
\text { WPG }(\%)=\frac{\left.\left(\mathrm{W}_{2}-\mathrm{W}_{1}\right) \mathrm{g}\right)}{\mathrm{W}_{1}(\mathrm{~g})} \times 100
$$

$\mathrm{W} 1$ and $\mathrm{W} 2$ are the initial dry weight and weight after immersion in water, respectively.

\section{Results and Discussion}

Water absorption property for hybrid-MDF showed a different trend in water absorption capability and this can be correlated to the strong dependence on the interface adhesion between fiber and the polymer matrix. Consequently, the hybrid system contains KF, which has higher content of cellulose and it makes a good hydrophilic source along with strong polar encapsulated polymer layer of hydrophobicity [11]. Hence higher water absorption corresponds to an increase in fiber loading because of fibers hydrophilicity, contributed to the hybrid-MDF system from both the fibers. Thus for hybrid system a constant increase in water absorption values with increased number of immersion days were observed while for KF-MDF and EFBMDF, after the longer immersion duration the values became almost constant. From the detailed water absorption studies of hybridMDF in the presence of ATH, a similar trend near to KF-MDF was observed as presented in Table (1) and Fig.(1). 
Table (1)

Water absorption of hybrid-MDF filled with 0, 5, 10 and $15 \%$ w/w ATH.

\begin{tabular}{||c||c||c||c||c||c||c||c|c||}
\hline Samples & D0 & D1 & D2 & D3 & D4 & D5 & D6 & D7 \\
\hline \hline Control hybrid-MDF & 2.01 & 3.58 & 3.96 & 4.07 & 4.09 & 4.10 & 4.11 & 4.12 \\
\hline \hline hybrid-MDF 5\% ATH & 2.01 & 3.47 & 3.85 & 3.94 & 3.99 & 4.03 & 4.05 & 4.06 \\
\hline \hline hybrid-MDF 10\% ATH & 2.03 & 3.41 & 3.80 & 3.88 & 3.92 & 3.96 & 3.96 & 3.98 \\
\hline \hline hybrid-MDF 15\% ATH & 2.04 & 3.43 & 3.71 & 3.79 & 3.81 & 3.84 & 3.86 & 3.86 \\
\hline
\end{tabular}

For unfilled hybrid-MDF, the water absorption values are much similar to unfilled KF-MDF [12] and immersing it to several days from D1 to D7 continuously exhibited a constant shift towards higher side was observed. The reason is due to the interaction of hydroxyl molecule from water medium with the hydroxyl groups from cellulosic and hemicellulose presented within the hybrid fiber structures [13]. There was a drop of $3.1 \%$ and up to D4 in the water absorption value when hybrid-MDF was supplemented with $5 \%$ ATH. The rise in water absorption on D4 was ascribed to the hybrid system's saturation point. Likewise, the water absorption rate with $5 \%$ ATH showed a change of: $42.1 \%, \mathrm{D} 2-10.9 \%$, D3 - $2.3 \%$, D4 - $1.3 \%$, D5 - $0.5 \%$, and last day $-0.2 \%$. Likewise, on raising the ATH loading concentration to $10 \%$ and $15 \%$, a similar pattern of change in water absorption was noted.
At the onset, the value dropped from D1 to D4. An incremental rise was noted from D4 to D7 continually. The rate of water absorption rose by $1.7 \%$ with $10 \%$ ATH loading in case of D1. Likewise, the rate of water absorption dropped by $0.6 \%$ when the hybrid-MDF was supplemented with $15 \%$ ATH. Thus, water absorption of hybrid-MDF can be said to be primarily dependent on the period of immersion, structural configuration, fillermatrix interaction, and the form of immersing medium. Structurally, the hybrid-MDF is consisted of mixed fiber systems at 65:35 fibers to pPU. Therefore, resulting water absorption will increase accordingly because the fibers are less likely to be fully encapsulated by the matrix of pPU $[14,15]$.

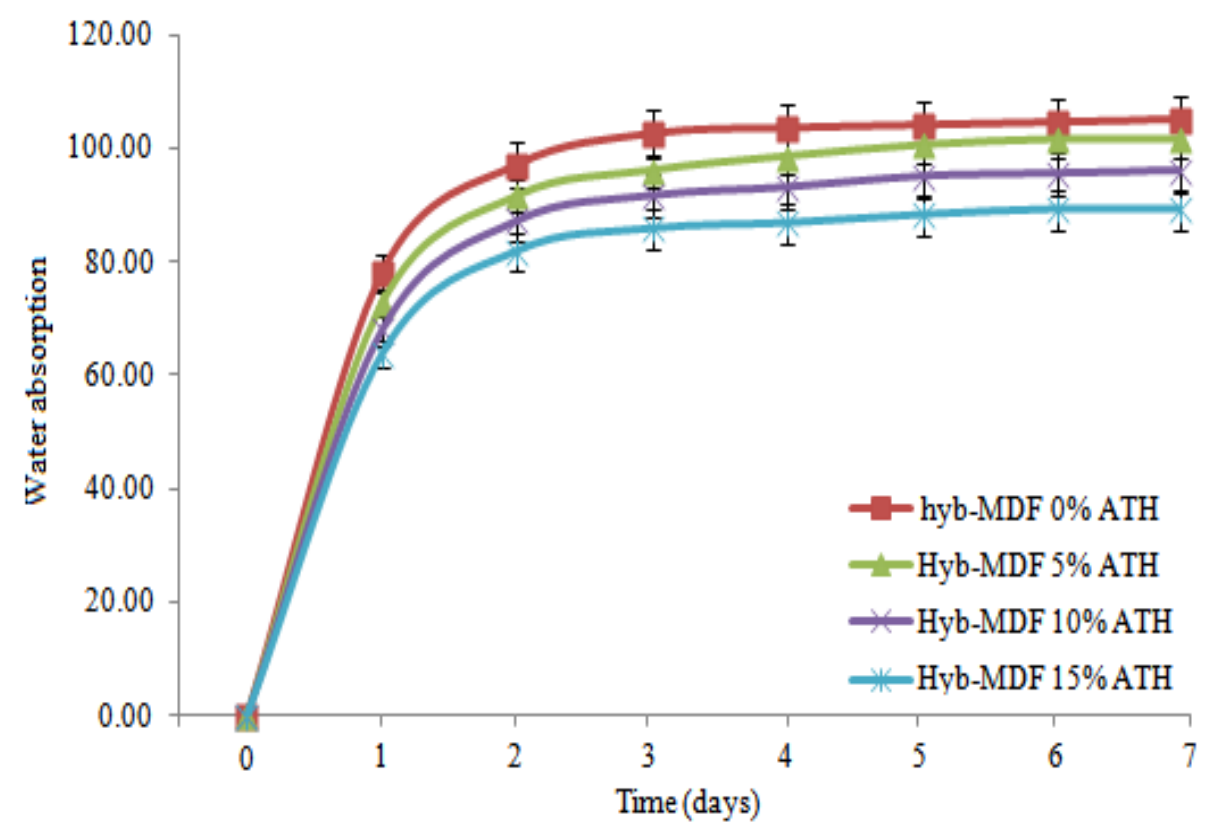

Fig.(1): Water absorption of hybrid-MDF filled with 0, 5, 10 and $15 \%$ w/w ATH. 


\section{Conclusion}

This present study revealed that filled Hybrid-MDF with ATH shows better water absorption compare to unfilled Hybrid-MDF. However, the changes in water rate absorption after D4 are very small but still in an increasing order which clearly signify the predominance of one fiber over the other besides the synergistic effect of fibers in combination with various loading concentration of ATH.

\section{Acknowledgements}

The principal researcher would like to thank the Department of chemistry and Faculty of Sciences, Bani Waleed University for facilities and services given. The authors wish to thank Polymer Research Centre, National university of Malaysia for all facilities offered and the Malaysia Ministry of Science, Technology and Innovation for finance and facilities given.

\section{References}

[1] Amin M., Anuar K., Haji Badri, K., PalmBased Bio- Composites Hybridized with Kaolinite. Journal of Applied Polymer Science, 105(5), 2488-2496, 2007.

[2] RedwanA., Haji Badri, K., Bahrum A., An Overview on Lignocellulosic Fibers Ienforced Polymer Composite Materials, Journal of Al-Nahrain University, 20(1), 25-31, 2017.

[3] Redwan M. A., Haji Badri K., Tarawneh, M. A., The Effect of Aluminium Hydroxide (Ath) on the Mechanical Properties and Fire Resistivity of Palm-Based Fibreboard Prepared by Pre-Polymerization Method. Advanced Materials Research, 287-292, 2015.

[4] Rowell R. M. Handbook of wood chemistry and wood composites. 2nd Ed, CRC press. Tylor and Francis Group. USA, 2012.

[5] Ye X. P., Julson, J., Kuo M., Womac A., Myers D., Properties of Medium Density Fiberboards Made from Renewable Biomass. Bioresource Technology, 98(5), 1077-1084, 2007.

[6] Halvarsson S., Edlund H., Norgren M., Properties of Medium-Density Fibreboard (Mdf) Based on Wheat Straw and
Melamine Modified Urea Formaldehyde (Umf) Resin. Industrial Crops and Products, 28(1), 37-46, 2008.

[7] Clemitson I. R., Castable Polyurethane Elastomers. Taylor and Francis. (New York): CRC Press, 2015.

[8] Wong C. S., Haji Badri K., Chemical Analyses of Palm Kernel Oil-Based Polyurethane Prepolymer. Scientific Research Publishing, (3), 78 - 86, 2012.

[9] Akgul M., Çamlibel O., Manufacture of Medium Density Fiberboard (Mdf) Panels from Rhododendron (R. Ponticum L.) Biomass. Building and Environment, 43(4), 438-443, 2008.

[10] Badri K. H., Redwan A. M., Effect of phosphite loading on the mechanical and fire properties of palm-based polyurethane. Sains Malaysiana, 39(5), 769-774, 2010.

[11] Stevulova N., Cigasova J., Purcz P., Schwarzova I., Kacik F., Geffert A., Water absorption behavior of hemp hurds composites. Materials, 8(5), 2243-2257, 2015.

[12] Redwan M. A., Haji Badri, K., Bahrum A., The mechanical characteristics of hybridised MDF from empty fruit bunch as well as kenaf following ATH treating and prepared by pre-polymerisation method. The 2016 UKM FST Postgraduate Colloquium. Published by AIP Publishing. 978-0-7354-1446-4, 2016.

[13] Topgaard D., \& Soderman O., Diffusion of water absorbed in cellulose fibers studied with 1H-NMR. Langmuir, 17(9), 26942702, 2011.

[14] Arib R., Sapuan S., Ahmad M., Paridah, M., Zaman, H. K. Mechanical Properties of Pineapple Leaf Fibre Reinforced Polypropylene Composites. Materials \& Design 27(5), 391-396, 2006.

[15] Acha B. A., Marcovich N. E, Reboredo M. M., Physical and Mechanical Characterization of Jute Fabric Composites. Journal of Applied Polymer Science, 98(2), 639-650, 2005. 